\title{
Long-Term Survival after Resection of Lung Metastases from Hepatocellular Cancer: Report of a Case and Review of the Literature
}

\author{
Sneha Chelimeda ${ }^{a}$ Teresa Bejarano $^{a}$ Robert Lowe $^{b}$ \\ Mahmoud Soliman ${ }^{c}$ Qing Zhao ${ }^{c}$ Kevan L. Hartshorn ${ }^{a}$

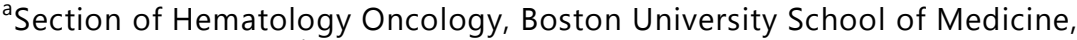 \\ Boston, Mass., USA; ${ }^{b}$ Section of Gastroenterology, Boston University School of Medicine, \\ Boston, Mass., USA; ${ }^{C}$ Department of Pathology, Boston University School of Medicine, \\ Boston, Mass., USA
}

\section{Keywords}

Hepatocellular cancer · Lung metastases · Hepatitis C · Metastasectomy ·

\begin{abstract}
Hepatocellular cancer (HCC) is increasing dramatically in incidence in Europe and the United States due mainly to the hepatitis $C$ epidemic and, to a lesser extent, increased body mass index of the population. In the fairly recent past, HCC was largely considered as untreatable due to detection mainly at late stages and lack of effective drugs for treatment. Several advances have led to changes in the prognosis of HCC. Screening of high-risk populations has allowed for earlier detection in some studies. If found at an early stage, liver transplantation not only cures the usual underlying cirrhosis but has cure rates for HCC in the range of $60 \%$ in recent series. Larger lesions can sometimes be cured by partial hepatic resection assuming the remaining liver is not too damaged to sustain liver functions after surgery. Vaccination for hepatitis B has led to reduction in the incidence of HCC. Significant improvements in antiviral treatments for both hepatitis $B$ and hepatitis $C$ may be having an impact on the incidence of HCC as well. It is still generally held that a finding of metastases precludes cure of HCC. We here report the case of a patient who presented with a large HCC in the context of
\end{abstract}


occult hepatitis $C$ infection. The primary tumor was resected. Over a year later, he developed a lung metastasis that was resected as well. He has not shown recurrence for 6 years since the metastasectomy. We review the recent literature on resection of lung metastases from HCC.

(c) 2016 The Author(s)

Published by S. Karger AG, Basel

\section{Case Report}

A 62-year-old male patient presented with a large left-sided liver mass $(11.3 \times 9.1 \times 10.5$ $\mathrm{cm}$, radiographically), which on biopsy proved to be a well-differentiated HCC (grade 1/4). There was portal fibrosis without bridging formation or cirrhosis in the adjacent liver (ISHAK fibrosis score 1-2/6). Liver function tests were normal at this time. The mass had a well-defined capsule which enhanced on equilibrium phase images. There was no evidence of hepatic or portal venous invasion. Several small lung nodules were seen on chest CT scan, the largest of which was $7 \mathrm{~mm}$ in the left lower lobe. This was removed by wedge resection and was found to have some benign changes with emphysematous and some nonnecrotizing granulomas and anthracosis. Based on this finding, it was decided to proceed with resection of the liver tumor. He then underwent a left hepatectomy which confirmed the diagnosis of HCC. Tumor was solitary, measuring $12 \mathrm{~cm}$ in the largest dimension, confined to the capsules with a negative resection margin (12 $\mathrm{mm}$ away). Tumor invasion in small vessels is also present. Tumor was staged as pT1 (however, based on the new AJCC 7th edition, tumor would be staged as pT2). There is no cirrhosis in the background liver. Approximately 13 months later, a 1.5-cm lingular nodule was noted on chest CT (enlarged from nodule seen 13 months prior in the same location). He underwent a left upper lobe wedge resection and complete lingular segmentectomy and the lingular nodule showed metastatic HCC. In the 6 years since resection of this metastasis, there has been no evidence of recurrence on CT scans of the chest, liver MRI or $\alpha$-fetoprotein testing. Two years after resection of the lung metastasis, he underwent a course of pegylated interferon and ribavirin lasting 1 year and teleprevir lasting 6 months for his hepatitis genotype 1 infection. His viral load has remained undetectable since completion of therapy. The patient remains in good general health now working full time and exercising regularly. His liver function tests have remained normal. Figure 1 shows pathological findings from the primary liver resection and the lung metastasis. Figure 2 shows results of liver MRI pre- and post-resection of the primary tumor and of CT scan pre- and post-resection of the lung metastasis.

\section{Discussion}

The most frequent site of distant spread from HCC is the lungs. Most of the literature regarding resection of lung or other metastases in patients with HCC comes from Asia where there are high incidences of HCC. A recent review by Zhou et al. [1] summarized reported series of patients undergoing pulmonary metastasectomy for HCC. Some of these involved patients who developed the metastases after liver transplantation and other were in patients who had the primary cancer resected (as in our patient). The median survival in these series overall varied between 10.7 and 77 months (mean 33.2 months) and the 5-year overall survival varied from 11.5 to $75 \%$ (median $36 \%$ ). Due to small numbers in most series, determination of prognostic variables has been challenging $[2,3]$. Some suggested favorable features include longer disease-free interval prior to development of the metastases, low $\alpha$-feto- 
protein level, control of the primary tumor, presence of only 1 or 2 metastases, lack of extrapulmonary metastases, and degree of tumor differentiation $[1,4,5]$. Note, however, that one series found good 2- and 3-year survivals even after resection of multiple pulmonary metastases [6]. Our case fits with many of these favorable variables including control of the primary, well-differentiated tumor, low $\alpha$-fetoprotein level, a 13-month disease-free interval between the primary tumor resection and the metastasectomy, and presence of only one metastasis.

In the reported series summarized by Zhou et al. [1], the majority of cases were associated with hepatitis B infection ( $75 \%)$ as compared to $\sim 25 \%$ of cases with hepatitis C infection. The results were not broken down according to outcomes between these two groups. Recent evidence suggests that control of hepatitis B infection improves postoperative prognosis in HCC [7]. In addition, a recent study found that lower hepatitis C viral load predicts better long-term outcome in patients who have resection of HCC [8]. Up until recently, treatment of hepatitis $\mathrm{C}$ infection was much more challenging than treatment of hepatitis $\mathrm{B}$; however, new regimens are much better tolerated and have broader coverage of diverse strains of hepatitis $\mathrm{C}$. These regimens have improved the tolerance and success of treatment greatly. Our case is of note since he had hepatitis $\mathrm{C}$ virus and was able to undergo effective treatment with one of the newer regimen of antivirals. Further improvements have already been made in hepatitis $\mathrm{C}$ virus treatment since our patient was treated. Perhaps these treatments will alter the risk HCC development or recurrence. Future studies are likely to address this question.

Our case is illustrative of improving the outlook for HCC and indicates that metastasectomy is worthy of consideration in patients with a resectable or resected primary tumor. His survival is comparable to the longest median survival reported in the literature. Our case and the recent literature appears to support a more aggressive approach to treatment of patients who have HCC and lung metastases, since outcomes are comparable to results with resection of lung metastases in colorectal cancer [9-11]. It is our impression that practitioners in the United States and Europe may be less aware of this literature. Of interest, if the initial lung biopsy had been positive in our patient, our plan was to not proceed with resection of the primary tumor.

\section{Statement of Ethics}

The authors have no ethical conflicts to disclose.

\section{Disclosure Statement}

The authors declare no conflicts of interest.

\section{References}

1 Zhou YM, Zhang XF, Yu F, Liu XB, Wu LP, Li B, et al: Efficacy of surgical resection for pulmonary metastases from hepatocellular carcinoma. Med Sci Monit 2014;20:1544-1549.

-2 Kow AW, Kwon CH, Song S, Shin M, Kim JM, Joh JW: Risk factors of peritoneal recurrence and outcome of resected peritoneal recurrence after liver resection in hepatocellular carcinoma: review of 1,222 cases of hepatectomy in a tertiary institution. Ann Surg Oncol 2012;19:2246-2255. 
-3 Kwon JB, Park K, Kim YD, Seo JH, Moon SW, Cho DG, et al: Clinical outcome after pulmonary metastasectomy from primary hepatocellular carcinoma: analysis of prognostic factors. World J Gastroenterol 2008;14:5717-5722.

4 Ohba T, Yano T, Yoshida T, Kawano D, Tsukamoto S, Shoji F, et al: Results of a surgical resection of pulmonary metastasis from hepatocellular carcinoma: prognostic impact of the preoperative serum alpha-fetoprotein level. Surg Today 2012;42:526-531.

5 Hau HM, Schmelzle M, Benzing C, Ascherl R, Tautenhahn HM, Gabelein G, et al: Pulmonary metastasectomy for metastasized hepatocellular carcinoma after liver resection and liver transplantation: a single center experience. Z Gastroenterol 2016;54:31-39.

-6 Mizuguchi S, Nishiyama N, Izumi N, Tsukioka T, Komatsu H, Iwata T, et al: Clinical significance of multiple pulmonary metastasectomy for hepatocellular carcinoma. World J Surg 2016;40:380-387.

7 Yin J, Li N, Han Y, Xue J, Deng Y, Shi J, et al: Effect of antiviral treatment with nucleotide/nucleoside analogs on postoperative prognosis of hepatitis B virus-related hepatocellular carcinoma: a two-stage longitudinal clinical study. J Clin Oncol 2013;31:3647-3655.

-8 Shindoh J, Hasegawa K, Matsuyama Y, Inoue Y, Ishizawa T, Aoki T, et al: Low hepatitis C viral load predicts better long-term outcomes in patients undergoing resection of hepatocellular carcinoma irrespective of serologic eradication of hepatitis C virus. J Clin Oncol 2013;31:766-773.

$\$ 9$ Dzian A, Uhnak M, Hamzik J: Surgical treatment of lung metastases of colorectal carcinoma - survival and prognostic factors. Klin Onkol 2015;28:345-351.

10 Mineo TC, Ambrogi V, Tonini G, Bollero P, Roselli M, Mineo D, et al: Long-term results after resection of simultaneous and sequential lung and liver metastases from colorectal carcinoma. J Am Coll Surg 2003;197:386-391.

$\checkmark 11$ Ike H, Shimada H, Ohki S, Togo S, Yamaguchi S, Ichikawa Y: Results of aggressive resection of lung metastases from colorectal carcinoma detected by intensive follow-up. Dis Colon Rectum 2002;45:468473; discussion 473-475. 


\section{Case Reports in Oncology}
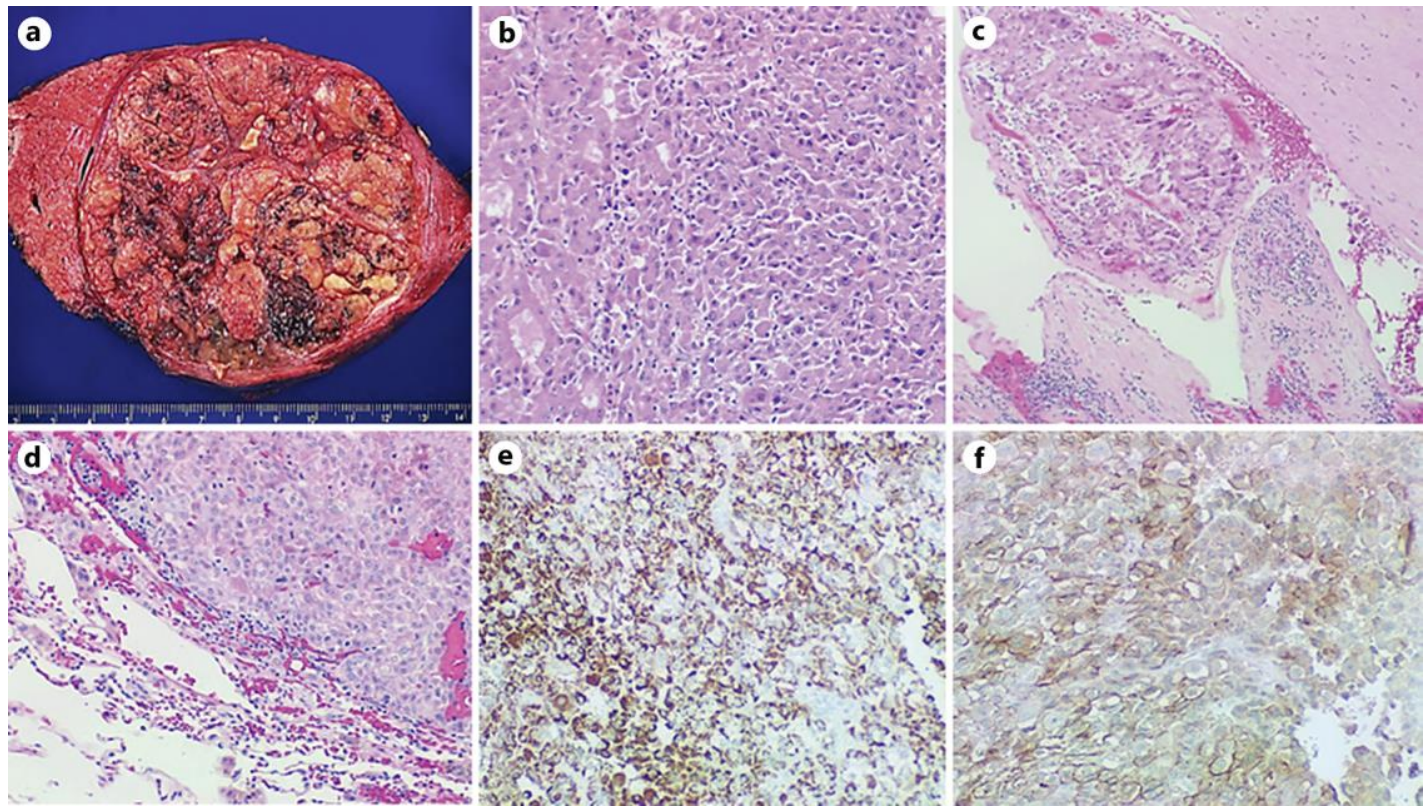

Fig. 1. Histopathology of the primary tumor and surrounding liver and of the pulmonary metastasis. A cross-section of the primary HCC showing variegated cut surface, fibrous septae with hemorrhage and necrosis and surrounding unremarkable liver parenchyma (a); tumor is well differentiated forming trabecular and pseudoglandular patterns (b, $\times 100$ magnification) with the presence of vascular space invasion (c, $\times 40$ magnification). The H\&E and immunohistochemical stains for pulmonary metastatic HCC show similar histopathology of the primary tumor ( $d, \times 100$ magnification), confirmed by positive stains for hepatocyte-specific antigen and glypican -3 (e and f, respectively, $\times 100$ magnification each). 


\section{Case Reports in Oncology}

www.karger.com/cro

Chelimeda et al.: Long-Term Survival after Resection of Lung Metastases from Hepatocellular Cancer: Report of a Case and Review of the Literature

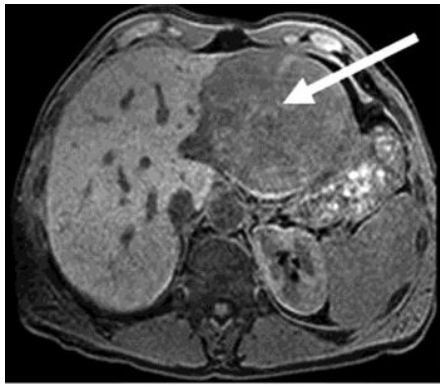

$\mathrm{MRI}$ at diagnosis

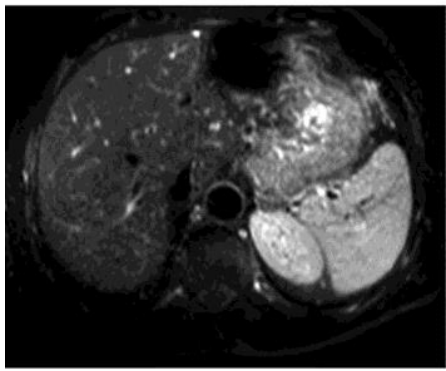

MRI post resection

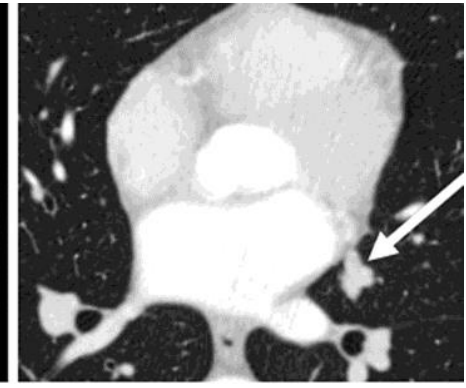

Lung metastasis

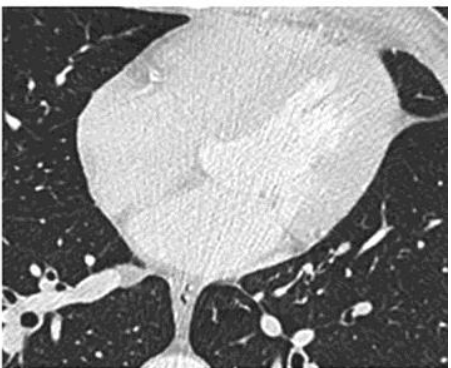

CT Post resection

Fig. 2. Imaging of liver and lung pre- and post-resection. The left panels show MRI at the time of diagnosis (the arrow indicates the large HCC) and post-resection. The right panels show CT scans at the time of diagnosis of the lung metastasis (indicated by an arrow) and post-resection. 